\title{
TIME-HOMOGENEOUS DIFFUSIONS WITH A GIVEN MARGINAL AT A RANDOM TIME
}

\author{
Alexander M.G. Cox ${ }^{1}$, David Hobson $^{2}$ And Jan ObŁó ${ }^{3}$
}

\begin{abstract}
We solve explicitly the following problem: for a given probability measure $\mu$, we specify a generalised martingale diffusion $\left(X_{t}\right)$ which, stopped at an independent exponential time $T$, is distributed according to $\mu$. The process $\left(X_{t}\right)$ is specified via its speed measure $m$. We present two heuristic arguments and three proofs. First we show how the result can be derived from the solution of [Bertoin and Le Jan, Ann. Probab. 20 (1992) 538-548.] to the Skorokhod embedding problem. Secondly, we give a proof exploiting applications of Krein's spectral theory of strings to the study of linear diffusions. Finally, we present a novel direct probabilistic proof based on a coupling argument.
\end{abstract}

Mathematics Subject Classification. 60G40, 60J60.

Received November 27, 2009. Revised February 25, 2010.

\section{INTRODUCTION}

We are interested in the following general problem: suppose $\mu$ is a given distribution on $\mathbb{R}$, and suppose $T$ is a (possibly random) time. Find a time-homogeneous martingale diffusion, $\left(X_{t}\right)$, independent of $T$, for which $X_{T}$ is distributed according to $\mu$. In particular, when $\mu$ is regular enough, we want to specify a function $\sigma: \mathbb{R}_{\rightarrow} \rightarrow \mathbb{R}_{+}$ such that

$$
X_{T}=\int_{0}^{T} \sigma\left(X_{s}\right) \mathrm{d} W_{s} \sim \mu,
$$

where $\left(W_{t}\right)$ is a Brownian motion. The process $\left(X_{t}\right)$ is a diffusion on natural scale described by its speed measure $m(\mathrm{~d} x)=\sigma(x)^{-2} \mathrm{~d} x$. When $\mu$ is less regular, the interpretation of $m$ as a speed measure remains valid, but $m$ may no longer have a density. In this case $X$ becomes a generalised or gap diffusion.

In this paper we present a solution to the problem in the case where $T$ is distributed exponentially with parameter 1. Somewhat surprisingly, not only does this problem always have a solution but also the solution is fully explicit. This can be seen both using a probabilistic and an analytic approach. More precisely, in Section 3 we exploit the general solution to the Skorokhod embedding problem of Bertoin and Le Jan [1].

\footnotetext{
Keywords and phrases. Time-homogeneous diffusion, generalised diffusion, exponential time, Skorokhod embedding problem, Bertoin-Le Jan stopping time.

1 Dept. of Mathematical Sciences, University of Bath, Bath BA2 7AY, UK. A.M.G.Cox@bath.ac.uk;

web: www.maths.bath.ac.uk/ mapamgc/

2 Department of Statistics, University of Warwick, Coventry CV4 7AL, UK. D.Hobson@warwick.ac.uk;

web: www.warwick.ac.uk/go/dhobson/

3 Mathematical Institute, University of Oxford, Oxford OX1 3LB, UK. obloj@maths.ox.ac.uk;

web: www.maths.ox.ac.uk/ obloj/
} 
This construction is essentially based on the theory of additive functionals for (nice) Markov processes. Then, in Section 4, we present a second proof based on the theory of generalised diffusions as presented in [14]. This is a more analytic approach which hinges on duality between generalised diffusions and strings and uses Krein's spectral theory of strings.

Both of the above proofs exploit deep known results. In the final section, we present a direct proof from first principles. First we prove that the problem has a solution. We do this by writing $X$ as a time-changed Brownian motion, $X_{t}=B_{A_{t}}$ and hence $A_{T}$ is a solution to the Skorokhod embedding in $B$ : that is, $B_{A_{T}}$ has distribution $\mu$. Our proof relies on a specific coupling of time-changes $A_{t}$ for different processes $X$. Furthermore, the interpretation in terms of stopping times for $B$ gives an intuitive justification for the explicit formula for $X$ (i.e. $\sigma$ in Eq. (1.1)).

\subsection{Motivation}

Our original goal was to solve the problem for the case where the time $T$ is a fixed, positive constant. Then, a time-homogeneous diffusion with $T$-marginal $\mu$ is in some sense the canonical, and perhaps simplest, process consistent with a given (single) marginal. For the problem with fixed $T$ and under sufficient smoothness conditions, Jiang and Tao [11] have proposed a relaxation scheme for calculating the diffusion co-efficient. However, to the best of our knowledge, existence and uniqueness for the problem with a general measure $\mu$ remain open. One application of this result would be to mathematical finance: the traded prices of call options with maturity $T$ can be used to infer the marginal distribution of the underlying asset under the pricing measure - note also that the price process $\left(S_{t}\right)$, suitably discounted, is a martingale under this measure - so that the solution of the problem for fixed $T$ would give a canonical candidate price process consistent with market data. Other time-inhomogeneous processes exist with the correct time $T$ marginals $(c f .[3,7,17])$, and the problem of finding examples, is related to the Skorokhod embedding problem. For further discussion of the Skorokhod embedding problem, and the connection to finance and model-independent hedging of derivatives, see Obłój [19] or Hobson [9].

Applications of the problem with $T$ exponentially distributed are discussed in a recent paper of Carr [2]. Carr proposes modelling the stock price process as a time-homogeneous diffusion time-changed by an independent gamma subordinator: $S_{t}=X_{\gamma_{t}}$. The clock is normalised so that $T=\gamma_{t^{*}}$ has an exponential distribution, where $t^{*}$ is now the maturity of options whose prices are known, so that Carr [2] effectively considers the same problem as the present paper. His approach is to use forward-backward Kolmogorov equations combined with Laplace transform properties. He is able to derive explicitly $\sigma$ in equation (1.1), although he only considers $\mu$ with positive density and does not prove general existence or uniqueness results.

\subsection{Heuristics}

We close the introduction with a couple of heuristic derivations of our result; one probabilistic and one analytic $^{1}$. In both cases we assume that we are in the "nice" case where the target law $\mu$ is centred and has a density. Later, our goal will be to provide a unified treatment which covers general probability measures $\mu$ with a finite first moment.

Suppose that the martingale $\left(X_{t}\right)$ is sufficiently regular that it can be represented as the solution of the SDE $\mathrm{d} X_{t}=\sigma\left(X_{t}\right) \mathrm{d} W_{t}$, with initial condition $X_{0}=0$, and that $\mu$ has density $\rho$. If $\left(X_{t}\right)$ stopped at an independent, rate 1 , exponential time $T$ is such that $X_{T} \sim \mu$ then we must have

$$
\mathbb{P}(\text { stop in }(x, x+\mathrm{d} x))=\mathbb{E}(\text { time in }(x, x+\mathrm{d} x)) \times(\text { Rate of stopping at } x) .
$$

By hypothesis the first term is $\rho(x) \mathrm{d} x$ and the last term is unity. For the middle term, by Itô's formula,

$$
f\left(X_{T}\right)-f\left(X_{0}\right)=\int_{0}^{T} f^{\prime}\left(X_{s}\right) \mathrm{d} X_{s}+\frac{1}{2} \int_{0}^{T} f^{\prime \prime}\left(X_{s}\right) \sigma^{2}\left(X_{s}\right) \mathrm{d} s,
$$

\footnotetext{
${ }^{1}$ We thank the referee for outlining the second argument to us.
} 
so that setting $f(z)=|z-x|$ and taking expectations we obtain

$$
\mathbb{E}\left[\left|X_{T}-x\right|-|x|\right]=\sigma(x)^{2} \mathrm{~d} x \mathbb{E}\left[\int_{0}^{T} \delta_{x}\left(X_{s}\right) \mathrm{d} s\right]
$$

where $\delta_{x}$ is the delta function at $x$. Hence $\sigma$ should be taken to solve

$$
\sigma(x)^{2} \rho(x)=\mathbb{E}\left[\left|X_{T}-x\right|-|x|\right] .
$$

Now we give an argument via resolvents. Let $V$ denote the one-resolvent of $\left(X_{t}\right)$, so that $V f(x)=$ $\mathbb{E}^{x}\left[\int_{0}^{\infty} e^{-s} f\left(X_{s}\right) \mathrm{d} s\right]$, and write $\mathcal{G}$ for the generator of $\left(X_{t}\right)$, so that $\mathcal{G} f(x)=\sigma(x)^{2} f^{\prime \prime}(x) / 2$. Then we want to choose $\left(X_{t}\right)$, or equivalently $V$ or $\mathcal{G}$, such that for a sufficiently wide class of $f$,

$$
V f(0)=\langle f, \rho\rangle,
$$

where $\langle g, h\rangle=\int_{\mathbb{R}} g(x) h(x) \mathrm{d} x$. The resolvent equation is $V(f-\mathcal{G} f)=f$ and since $V$ and $\mathcal{G}$ commute we have $f=V f-\mathcal{G} V f$. Writing $h=V f$, and $\mathcal{G}^{*}$ for the adjoint operator, equation (1.3) becomes $\left\langle h, \delta_{0}\right\rangle=h(0)=$ $\langle h, \rho\rangle-\langle\mathcal{G} h, \rho\rangle=\langle h, \rho\rangle-\left\langle h, \mathcal{G}^{*} \rho\right\rangle$. Since $h$ is a test function, we have that $\rho$ solves

$$
\rho-\frac{1}{2}\left(\sigma^{2} \rho\right)^{\prime \prime}=\delta_{0}
$$

and $\sigma^{2} \rho$ is the second anti-derivative of $2\left(\rho-\delta_{0}\right)$, from which we recover equation (1.2).

\section{Generalised (Gap) Diffusions}

We recall the classical construction of a generalised diffusion. Let $m_{i}:[0, \infty] \rightarrow[0, \infty]$ be non-decreasing and right-continuous with $m_{i}(\infty)=\infty$ and $\ell_{i}=\sup \left\{x: m_{i}(x)<\infty\right\}>0, i=1,2$. Assume further that $m_{2}(0+)=0$. Then $\mathrm{d} m_{i}$ are well defined measures and we can define a measure $m$ on $\mathbb{R}$ by

$$
m(\mathrm{~d} x)= \begin{cases}\mathrm{d} m_{1}(x) & \text { for } x \in[0, \infty) \\ \mathrm{d} \check{m}_{2}(x) & \text { for } x \in(-\infty, 0)\end{cases}
$$

where $\mathrm{d} \check{m}_{2}$ is the image of $\mathrm{d} m_{2}$ under $x \rightarrow-x$. Naturally, $\ell_{i}$ can be defined directly from $m$ and we write $\ell_{-}=\ell_{-}(m)=-\ell_{2}$ and $\ell_{+}=\ell_{+}(m)=\ell_{1}$.

Consider $\left(B_{t}, \mathbb{P}^{x_{0}}\right)$ a one-dimensional Brownian motion defined on $\left(\Omega, \mathcal{F},\left(\mathcal{F}_{t}\right)\right)$, with $B_{0}=x_{0}, \mathbb{P}^{x_{0}}$-a.s. We assume $\mathcal{F}_{0}$ is rich enough to support random variables independent of $B$. Let $\left(L_{t}^{x}\right)$ be the jointly continuous version of the local time of $\left(B_{t}\right)$. We adopt here the classical Itô-McKean normalisation in which $\left|B_{t}-x\right|-L_{t}^{x}$ is a martingale. Put $\Phi_{t}=\int_{\mathbb{R}} L_{t}^{x} m(\mathrm{~d} x)$ and let $\left(A_{t}\right)$ be the right-continuous inverse of $\left(\Phi_{t}\right)$. Then

$$
\left(X_{t}, \mathbb{P}^{x_{0}}\right), \quad X_{t}:=B_{A_{t}}, \quad \text { for } t \leq \zeta=\inf \left\{t \geq 0: X_{t} \notin\left(\ell_{-}, \ell_{+}\right)\right\}
$$

is a time-change of a Brownian motion and hence a strong Markov process living on $\operatorname{supp}(m)$. It is called a generalised diffusion (on natural scale) corresponding to the measure $m$. It has also been called a gap diffusion in [13]. Note that, due to our normalisation, the local time $L_{t}^{x}$ is twice the local time in [14]. In consequence $\left(X_{t}\right)$ is a generalised diffusion corresponding to measure $2 m$ in the notation of Kotani and Watanabe [14]. As an example, in this paper Brownian motion is a diffusion with speed measure equal to the Lebesgue measure and not twice the Lebesgue measure as in [14].

In order to understand better the relationship between features of $m$ and the behaviour of $X$ we discuss two important classes, firstly where $m$ has a positive density, and secondly where $m$ only charges points. 
Suppose first that $0<m([a, b])<\infty$ for any $\ell_{-}<a<b<\ell_{+}$. Then $\left(X, \mathbb{P}^{x}\right)$ is a regular diffusion on $^{2}$ $I=\left[\ell_{-}, \ell_{+}\right]$with absorbing boundary points. $X$ is on natural scale and $m(\mathrm{~d} x)$ is simply its speed measure. We have $A_{t}=[X]_{t}$ and the measure $m$ can be recovered from $X$ as

$$
m(\mathrm{~d} x)=-\frac{1}{2} h_{J}^{\prime \prime}(\mathrm{d} x), \quad h_{J}:=\mathbb{E}^{x}\left[\inf \left\{t: X_{t} \notin J\right\}\right], J=[a, b] \subset I,
$$

since it can be shown that $h_{J}(x)$ is convex and, for $J \subset K$, as measures $h_{J}^{\prime \prime}=h_{K}^{\prime \prime}$ on $\operatorname{int}(J)$, see [20], Section V.47, for a detailed discussion. If further $m(\mathrm{~d} x)=\lambda(x) \mathrm{d} x$, with $\lambda$ bounded and uniformly positive on $I$, then $\left(X_{t}\right)$ solves

$$
\mathrm{d} X_{t}=\lambda\left(X_{t}\right)^{-1 / 2} \mathrm{~d} W_{t}, \quad t<\zeta,
$$

for a Brownian motion $\left(W_{t}\right)$. Equivalently the infinitesimal generator of $X$, when acting on functions supported on $I$, is $\mathcal{G}=\frac{1}{2 \lambda(x)} \frac{\mathrm{d}^{2}}{\mathrm{~d} x^{2}}=\frac{1}{2} \frac{\mathrm{d}^{2}}{\mathrm{~d} m \mathrm{~d} x}$. Note that then $A_{t}=[X]_{t}=\int_{0}^{t} \lambda\left(X_{s}\right)^{-1} \mathrm{~d} s$ and it can be verified directly from the occupation time formula that $\Phi_{t}^{-1}=[X]_{t}$ :

$$
\begin{aligned}
\Phi_{[X]_{t}} & =\int_{\mathbb{R}} L_{[X]_{t}}^{x} m(\mathrm{~d} x)=\int_{\mathbb{R}} L_{[X]_{t}}^{x} \lambda(x) \mathrm{d} x \\
& =\int_{0}^{[X]_{t}} \lambda\left(B_{s}\right) \mathrm{d} s=\int_{0}^{t} \lambda\left(B_{[X]_{u}}\right) \mathrm{d}[X]_{u}=\int_{0}^{t} \lambda\left(X_{u}\right) \mathrm{d}[X]_{u}=t .
\end{aligned}
$$

From the above discussion we see that the regions where $m$ has more mass correspond to the regions where $\left(X_{t}\right)$ moves more slowly (and thus spends more time).

By a natural extension of the above analysis, if $m$ has zero mass in an interval, then since $\left(X_{t}\right)$ lives on the support of $m$, this interval is not visited by $\left(X_{t}\right)$ at all. Conversely, if $m(\{a\})>0$ (and for every neighbourhood $U$ of $a, m$ charges $U \backslash\{a\})$, then $a$ is a sticky point for $X$ : started in $a,\left(X_{u}: u \leq t\right)$ spends a positive time in $a$ even though it exits $a$ instantaneously.

It remains now to understand the role of isolated atoms in $m$. Consider $m=\sum_{i=1}^{N} \beta_{i} \delta_{a_{i}}$ for $a_{1}<\ldots<a_{N}$, $\beta_{i}>0$ and $\beta_{1}=\beta_{N}=\infty$. Then $X$ is a continuous-time Markov chain living on $\left\{a_{1}, \ldots, a_{N}\right\}$, stopped upon hitting $a_{1}$ or $a_{N}$. From the construction it follows that $X$ can only jump to nearest neighbours, i.e. from $a_{i}$ it can jump to $a_{i-1}, a_{i+1}$, and the probabilities of these follow instantly from the fact that $\left(X_{t}\right)$ is a martingale. The time spent in $a_{i}, 1<i<N$, before $\left(X_{t}\right)$ jumps to a next point, has an exponential distribution with mean

$$
\beta_{i} \mathbb{E}^{a_{i}}\left[L_{H_{a_{i-1}, a_{i+1}}^{a_{i}}}\right]=2 \beta_{i} \frac{\left(a_{i+1}-a_{i}\right)\left(a_{i}-a_{i-1}\right)}{a_{i+1}-a_{i-1}},
$$

where $H_{a, b}$ is the first hitting time of $\{a, b\}$ for $\left(B_{t}\right)$. (This is an example of the more general formula (for $a<x \wedge y \leq x \vee y<b)$

$$
\mathbb{E}^{x}\left[L_{H_{a} \wedge H_{b}}^{y}\right]=2 \frac{(x \wedge y-a)(b-x \vee y)}{b-a}
$$

for expected values of Brownian local times.) This completes the description of $X$. We see that an isolated atom in $a_{i}$ has an effect of introducing a holding time in that point for $X$, with mean proportional to $m\left(\left\{a_{i}\right\}\right)$.

\footnotetext{
${ }^{2}$ i.e. $\mathbb{P}^{x}\left(H_{y}<\infty\right)>0$ for all $x, y \in I$, where $H_{y}=\inf \left\{t>0: X_{t}=y\right\}$.
} 


\section{MAIN RESUlt AND PROBABILISTIC PROOF}

Having recalled generalised diffusions we can now state the main result of our paper. In this section we provide a proof rooted in probabilistic arguments while in the next section we describe a more analytical approach. For a probability measure $\mu$ we let $\ell_{-}^{\mu}$ and $\ell_{+}^{\mu}$ denote respectively the lower and the upper bounds of its support.

Theorem 3.1. Let $\mu$ be a probability measure, $\int|x| \mu(\mathrm{d} x)<\infty, \int x \mu(\mathrm{d} x)=x_{0}$ and let $u_{\mu}(x)=\int_{\mathbb{R}}|x-y| \mu(\mathrm{d} y)$. Define a measure $m$ by

$$
\begin{array}{ll}
m(\mathrm{~d} x)=\frac{\mu(\mathrm{d} x)}{u_{\mu}(x)-\left|x-x_{0}\right|} & \text { for } x \in\left(\ell_{-}^{\mu}, \ell_{+}^{\mu}\right), \\
m\left(\left[y, x_{0}\right)\right)=m\left(\left[x_{0}, x\right]\right)=\infty & \text { for } y \leq \ell_{-}^{\mu} \leq \ell_{+}^{\mu} \leq x .
\end{array}
$$

Let $\left(X_{t}\right)$ be the generalised diffusion associated with $m$ and $T$ be an $\mathcal{F}_{0}$-measurable $\mathbb{P}^{x_{0}}$-exponential random variable independent of $\left(X_{t}\right)$. Then, under $\mathbb{P}^{x_{0}}, X_{T} \sim \mu$ and $\left(X_{t \wedge T}\right)$ is a uniformly integrable martingale.

Proof. It suffices to prove the theorem for $x_{0}=0$ as the general case follows by a simple shift in space. Assume in first instance that $\operatorname{supp}(\mu) \subset(-N, N)$. Consider the following process $\left(Y_{t}\right)$ : it takes values in $(-N, N) \cup\{\star\}$, with $\star$ added as an isolated point. $Y$ starts in $\star$ which is a holding point with parameter 1 . On exit from $\{\star\}$ at time $\rho_{\star}$, the process behaves as $B$ under the measure $\mathbb{P}^{0}$, so that $Y_{\rho_{\star}+t}=B_{t}$, until exit from $(-N, N)$ when $Y$ jumps back to $\star$. In this way $\left(Y_{t}\right)$ is a recurrent strong Markov process with $\star$ as its regular starting point. Write $\tilde{\mathbb{P}}^{x}$ for the probability measure associated with the process $Y_{t}$ started at $x$, noting that for all $\tilde{\mathbb{P}}^{x}$, the path jumps from $\star$ to 0 . We make explicit the Bertoin and Le Jan solution [1] to the Skorokhod embedding problem of $\mu$ in $Y$.

Let $\tau_{\star}=\inf \left\{t>\rho_{\star}: Y_{t}=\star\right\}=\rho_{\star}+\inf \left\{t>0: B_{t} \notin(-N, N)\right\}=: \rho_{\star}+H$. The process $\left(Y_{t}\right)$ admits a family of local times $\left(L_{t}^{a}(Y)\right)$. We simply have $L_{t}^{a}(Y)=L_{t-\rho_{\star}^{a}}^{a},|a|<N$ and $L_{t}^{\star}(Y)=L_{\rho_{\star}}^{\star}(Y)$ for $\rho_{\star} \leq t<\tau_{\star}$. This last quantity is exponentially distributed and independent of $\left(B_{t}\right)$. It follows from equation $(2.5)$ that

$$
\begin{aligned}
& \tilde{\mathbb{E}}^{\star}\left[L_{\tau_{\star}}^{a}(Y)\right]=\mathbb{E}^{0}\left[L_{H}^{a}\right]=N-|a|, \quad|a|<N \\
& \tilde{\mathbb{E}}^{x}\left[L_{\tau_{\star}}^{a}(Y)\right]=\mathbb{E}^{x}\left[L_{H}^{a}\right]=\frac{(a \wedge x+N)(N-a \vee x)}{N},|a|<N,|x|<N .
\end{aligned}
$$

The invariant measure $\nu$ for $Y$, displayed in equation (1) in [1], acts by

$$
\begin{aligned}
\int f \mathrm{~d} \nu & =\tilde{\mathbb{E}}^{\star}\left[\int_{\rho_{\star}}^{\tau_{\star}} f\left(Y_{s}\right) \mathrm{d} s\right]+f(\star)=\mathbb{E}^{0}\left[\int_{0}^{H} f\left(B_{u}\right) \mathrm{d} u\right]+f(\star) \\
& =\int_{-N}^{N} f(a) \mathbb{E}^{0}\left[L_{H}^{a}\right] \mathrm{d} a+f(\star)=\int_{-N}^{N} f(a)(N-|a|) \mathrm{d} a+f(\star) .
\end{aligned}
$$

Consider a finite positive measure $\xi$ on $(-N, N)$ and a positive continuous additive functional $F_{t}=\int L_{t}^{a}(Y) \xi(\mathrm{d} a)$. The Revuz measure $\chi$ of $F$ is then given by

$$
\int f \mathrm{~d} \chi=\frac{1}{t} \tilde{\mathbb{E}}^{\nu}\left[\int_{0}^{t} f\left(Y_{s}\right) \mathrm{d} F_{s}\right]=\frac{1}{t} \int f(a) \tilde{\mathbb{E}}^{\nu}\left[L_{t}^{a}(Y)\right] \xi(\mathrm{d} a)=\int f(a)(N-|a|) \xi(\mathrm{d} a)
$$


so that $\xi(d a)=\chi(d a) /(N-|a|)$ and $\chi=\mu$ iff $\xi(\mathrm{d} a)=\frac{\mu(\mathrm{d} a)}{N-|a|}$. We proceed to compute $V_{\chi}$ and $\hat{V}_{\mu}$, as defined in [1]. We have $V_{\chi}(x)=\tilde{\mathbb{E}}^{x}\left[\int_{0}^{\tau_{\star}} \mathrm{d} F_{s}\right]$ and $\int \hat{V}_{\mu} \mathrm{d} \chi=\int V_{\chi} \mathrm{d} \mu$. Then, for $x \in(-N, N)$,

$$
\begin{aligned}
V_{\chi}(x) & =\int \mathbb{E}^{x}\left[L_{H}^{a}\right] \frac{\chi(\mathrm{d} a)}{N-|a|} \\
& =\int \frac{(N+a \wedge x)(N-a \vee x)}{N(N-|a|)} \chi(\mathrm{d} a),
\end{aligned}
$$

and it follows that

$$
\hat{V}_{\mu}(a)=\int \frac{(N+a \wedge x)(N-a \vee x)}{N(N-|a|)} \mu(\mathrm{d} x)=\frac{N-u_{\mu}(a)}{N-|a|} .
$$

Jensen's inequality grants us $u_{\mu}(a) \geq|a|$ and hence $\hat{V}_{\mu} \leq 1$ is bounded as required. Furthermore, from equation (3.5), for $0<a<N$

$$
\hat{V}_{\mu}(a) \geq \frac{\int_{a}^{N} u_{\mu}^{\prime}(x) \mathrm{d} x}{\int_{a}^{N} \mathrm{~d} x} \geq u_{\mu}^{\prime}(a+)=1-2 \mu((a, \infty)) \stackrel{a \uparrow N}{\rightarrow} 1
$$

since $\mu$ has support in $(-N, N)$. Hence the bound $\hat{V}_{\mu}(a) \leq 1$ is best possible.

We have

$$
\left(1-\hat{V}_{\mu}(a)\right)^{-1}=\frac{N-|a|}{u_{\mu}(a)-|a|}
$$

and the Bertoin-Le Jan stopping time [1] is given by

$$
T_{B L J}=\inf \left\{t \geq 0: \int_{0}^{t} \frac{N-\left|Y_{s}\right|}{u_{\mu}\left(Y_{s}\right)-\left|Y_{s}\right|} \mathrm{d} F_{s}>L_{t}^{\star}(Y)\right\}
$$

where $F_{t}=\int L_{t}^{a}(Y) \frac{\mu(\mathrm{d} a)}{N-|a|}$. The key result of Bertoin and Le Jan [1] is that $T_{B L J}$ solves the Skorokhod embedding problem for $\mu$ : i.e. $Y_{T_{B L J}} \sim \mu$. Recall that $X_{t}=B_{A_{t}}$ with $A_{t}$ the right-continuous inverse of $\Phi_{t}=\int L_{t}^{a} m(\mathrm{~d} a)$, where $m$ is as displayed in equation (3.1). By the corollary on p. 540 in $[1], \tilde{\mathbb{E}}^{\star}\left[L_{T_{B L J}}^{\star}(Y)\right]=1$ from which it follows that $T_{B L J}<\tau_{\star}$. This allows us to rewrite $T_{B L J}$ as

$$
\begin{aligned}
T_{B L J} & =\rho_{\star}+\inf \left\{t \geq 0: \int_{0}^{t} \frac{N-\left|B_{s}\right|}{u_{\mu}\left(B_{s}\right)-\left|B_{s}\right|} \mathrm{d} F_{s-\rho_{\star}}>L_{\rho_{\star}}^{\star}(Y)\right\} \\
& =\rho_{\star}+\inf \left\{t \geq 0: \int \frac{L_{t}^{a} \mu(\mathrm{d} a)}{u_{\mu}(a)-|a|}>L_{\rho_{\star}}^{\star}(Y)\right\} \\
& =\rho_{\star}+\inf \left\{t \geq 0: \Phi_{t}>L_{\rho_{\star}}^{\star}(Y)\right\}=\rho_{\star}+A_{L_{\rho_{\star}}(Y)} .
\end{aligned}
$$

Hence $B_{A_{T}}=X_{T} \sim \mu$, with $T=L_{\rho_{\star}}^{\star}(Y)$ an exponential random variable, as required. Note that by construction $\left(X_{t}\right)$ remains within the bounds of the support of $\mu$. In particular, uniform integrability of $\left(X_{t \wedge T}\right)$ follows from the fact that it is a bounded martingale.

Now consider an arbitrary $\mu$ and $m$ defined via equation (3.1). Note that $\ell_{-}^{\mu}=\ell_{-}(m)$ and $\ell_{+}^{\mu}=\ell_{+}(m)$. Naturally if $\mu$ has a bounded support then the previous reasoning applies, so suppose that $-\ell_{-}^{\mu}=\ell_{+}^{\mu}=\infty$. For $M>\left|u_{\mu}(0)\right|$, let $\mu_{M}$ be the measure on $\left[q_{M}^{-}, q_{M}^{+}\right] \cup\{-M, M\}$, centred in zero and with $u_{\mu_{M}}=u_{\mu}$ on $\left[q_{M}^{-}, q_{M}^{+}\right]$, and $u_{\mu_{M}} \leq u_{\mu}$. Note that this defines $q_{M}^{ \pm}$and $\mu_{M}$ uniquely, $q_{M}^{ \pm}$converge to the bounds of the support of $\mu$ as $M \rightarrow \infty, \mu_{M}=\mu$ on $\left(q_{M}^{-}, q_{M}^{+}\right), \mu\left(\left\{q_{M}^{ \pm}\right\}\right) \geq \mu_{M}\left(\left\{q_{M}^{ \pm}\right\}\right)$and $\mu_{M}$ converges weakly to $\mu$, see [4] for details. Let $A_{t}^{M}$ be the inverse of $\Phi_{t}^{M}=\int L_{t}^{x} m_{M}(\mathrm{~d} x)$, with $m_{M}$ given by equation (3.1) for $\mu_{M}$, and $X_{t}^{M}=B_{A_{t}^{M} \text {. Fix }}$ an exponential random variable $T$ independent of $\left(B_{t}\right)$. By the construction above (applied with $N=M+1$, 
so that the support of $\mu_{M}$ is contained in $\left.(-N, N)\right), X_{T}^{M} \sim \mu_{M}$. Observe that, since $m_{M}=m$ on $\left(q_{M}^{-}, q_{M}^{+}\right)$, we have $X_{t}^{M}=X_{t}$ for $t<\tau_{M}:=\inf \left\{t: X_{t} \notin\left(q_{M}^{-}, q_{M}^{+}\right)\right\}$. Since $\mathbb{P}\left(T \geq \tau_{M}\right) \rightarrow 0$, both $X_{T}^{M}$ and $X_{T} \mathbf{1}_{\left\{T<\tau_{M}\right\}}$ converge to the same limit in distribution as $M \rightarrow \infty$, and hence $X_{T} \sim \mu$.

To see that the process $X_{t \wedge T}$ is uniformly integrable, we note that this is equivalent to the process $B_{A_{t \wedge T}}$ being uniformly integrable. This is easy to see as a consequence of Proposition 18 of Cox [5], noting that since our laws $\mu_{n}, \mu$ are centred, uniform integrability and minimality are equivalent.

Finally, when only one of $\ell_{-}^{\mu}, \ell_{+}^{\mu}$ is infinite, say $\ell_{+}^{\mu}=\infty$, the procedure is analogous but we only truncate the support of $\mu$ on one side, i.e. we look at $\mu_{M}=\mu$ on $\left(q_{M}^{-}, \infty\right)$.

\section{An ANALYTIC PROOF}

In the previous section, we proved Theorem 3.1 using a probabilistic approach, characteristic of the work of Bertoin and Le Jan [1]. However, study of generalised diffusions can be seen as a probabilistic counterpart of the theory of strings, see [8]. Indeed the theory of strings and original results in [15] have played an important role in the study of fine properties of generalised diffusions including the Lévy measures of their inverse local times $^{3}$, lower bounds on the spectrum of their infinitesimal generator, asymptotics of their transition densities and first hitting times distributions see $[12-14,16]$. With this in mind our aim is to re-derive equation (3.1) using analytic methods. As in Section 1.2 we begin with an expression for the resolvent in terms of the speed measure of the diffusion, and then try to match this expression to the desired target law $\mu$.

Let $\left(X_{t}\right)$ be a generalised diffusion associated with a measure $m$ as in equation (2.1). Recall that in the notation of Kotani and Watanabe [14] $X$ is associated to measure $2 m$. Let $\phi, \psi, h_{ \pm}(\lambda), h(\lambda)$ and $u_{ \pm}$be defined as equations (3.1)-(3.4) in [14] (but with our normalisation of $m$ ):

$$
\begin{aligned}
\phi(x, \lambda) & = \begin{cases}1+2 \lambda \int_{0-}^{x+}(x-y) \phi(y, \lambda) m(\mathrm{~d} y) & : 0 \leq x<\ell_{+} \\
1+2 \lambda \int_{x-}^{0-}(y-x) \phi(y, \lambda) m(\mathrm{~d} y) & : \ell_{-}<x<0\end{cases} \\
\psi(x, \lambda) & = \begin{cases}x+2 \lambda \int_{0-}^{x+}(x-y) \psi(y, \lambda) m(\mathrm{~d} y) & : 0 \leq x<\ell_{+} \\
x+2 \lambda \int_{x-}^{0-}(y-x) \psi(y, \lambda) m(\mathrm{~d} y) & : \ell_{-}<x<0\end{cases} \\
h_{+}(\lambda) & =\int_{0}^{\ell_{+}} \frac{1}{\phi(x, \lambda)^{2}} \mathrm{~d} x=\lim _{x \uparrow \ell_{+}} \frac{\psi(x, \lambda)}{\phi(x, \lambda)} \\
h_{-}(\lambda) & =\int_{\ell_{-}}^{0} \frac{1}{\phi(x, \lambda)^{2}} \mathrm{~d} x=-\lim _{x \downarrow \ell_{-}} \frac{\psi(x, \lambda)}{\phi(x, \lambda)} \\
\frac{1}{h(\lambda)} & =\frac{1}{h_{+}(\lambda)}+\frac{1}{h_{-}(\lambda)} \\
u_{ \pm}(x, \lambda) & =\phi(x, \lambda) \mp \frac{\psi(x, \lambda)}{h_{ \pm}(\lambda)}
\end{aligned}
$$

These functions yield a direct representation (Eq. 3.5) therein:

$$
g_{\lambda}(x, y)=g_{\lambda}(y, x)=h(\lambda) u_{+}(x, \lambda) u_{-}(y, \lambda), \quad x \geq y,
$$

\footnotetext{
${ }^{3}$ Essentially Krein's theorem provides a bijection between the set of strings and the set of their spectral measures. This is equivalent with a bijection between generalised diffusions $\left(X_{t}\right)$ with $m_{2} \equiv 0$, reflected in zero, with the set of subordinators given by the inverse of the local time in zero of $X$.
} 
of the resolvent density $g_{\lambda}$, defined by

$$
\mathbb{E}^{x}\left[\int_{0}^{\zeta} \mathrm{e}^{-\lambda t} f\left(X_{t}\right) \mathrm{d} t\right]=\int_{\left(\ell_{-}, \ell_{+}\right)} 2 g_{\lambda}(x, y) f(y) m(\mathrm{~d} y), \quad x \in\left(-\ell_{-}, \ell_{+}\right)
$$

for continuous bounded functions $f$ on the support of $m$.

In what follows we take $\lambda=1$ and drop the $\lambda$ argument. We have $u_{ \pm}(0)=1$ and it can be checked independently (or deduced from Eqs. (4.1)-(4.2) above) that $u_{ \pm}$are non-negative with $u_{+}$non-increasing, and $u_{-}$non-decreasing. Further we have $u_{+}(x) \rightarrow 0$ as $x \rightarrow \ell_{+}$and $u_{-}(x) \rightarrow 0$ as $x \rightarrow \ell_{-}$. This is described in detail, in the case of standard diffusion processes, in Theorem 5.13.3 in [10] (note that our $u_{+}$is the solution $\underline{u}$ therein), see also [14] (p. 241) and [13] (p. 57). Furthermore, from their definitions, we have

$$
\begin{aligned}
& u_{+}^{\prime \prime}(\mathrm{d} x)=2 u_{+}(x) m(\mathrm{~d} x), \quad 0<x<\ell_{+}, \\
& u_{-}^{\prime \prime}(\mathrm{d} x)=2 u_{-}(x) m(\mathrm{~d} x), \quad \ell_{-}<x<0 \text {. }
\end{aligned}
$$

Assume for simplicity that $\mu$ is a centred probability measure: $x_{0}=0$, and put $U_{\mu}(x)=u_{\mu}(x)-|x|$. Then Theorem 3.1 is simply equivalent to showing that for $\lambda=1$ and $m$ given by equation $(3.1)$, that is $m(\mathrm{~d} x)=\frac{\mu(\mathrm{d} x)}{U_{\mu}(x)}$, we have $g_{1}(x, 0)=\frac{1}{2} U_{\mu}(x)$.

Observe now that $U_{\mu}(x)$ also solves equation (4.3) and $U_{\mu}(x) \rightarrow 0$ as $|x| \rightarrow \infty$. Given that $g_{\lambda}$ solves equation (4.1) it follows that $g(x)=g_{1}(0, x)=c U_{\mu}(x)$ for some constant $c$. It remains to show that $c=\frac{1}{2}$. For this we analyse the derivative at zero. On the one hand we have

$$
U_{\mu}^{\prime}(0-)-U_{\mu}^{\prime}(0+)=2 \mu([0, \infty))+2 \mu((-\infty, 0))=2
$$

and on the other hand

$$
g^{\prime}(0-)-g^{\prime}(0+)=h(\lambda)\left(u_{-}^{\prime}(0-)-u_{+}^{\prime}(0+)\right)=h(\lambda)\left(\frac{1}{h_{-}(\lambda)}+\frac{1}{h_{+}(\lambda)}\right)=1,
$$

from which it follows that $c=\frac{1}{2}$ as required.

To end this section we check that the boundary behaviour of $X$ at $\left\{\ell_{-}, \ell_{+}\right\}$is what we would expect it to be. Consider for example $\ell_{+}$. If $\mu\left(\left\{\ell_{+}\right\}\right)=0$ then we have

$$
\begin{aligned}
m\left(\left(0, \ell_{+}\right)\right) & =\int_{\left(0, \ell_{+}\right)} \frac{\mu(\mathrm{d} x)}{\int_{x}^{\ell_{+}}(y-x) \mu(\mathrm{d} y)} \geq \int_{\ell_{+}-\epsilon}^{\ell_{+}} \frac{\mu(\mathrm{d} x)}{\int_{x}^{\ell_{+}}(y-x) \mu(\mathrm{d} y)} \\
& \geq \frac{1}{\epsilon} \int_{\ell_{+}-\epsilon}^{\ell_{+}} \frac{\mu(\mathrm{d} x)}{\mu\left(\left[x, \ell_{+}\right]\right)}=\frac{1}{\epsilon}\left(\log \mu\left(\left[\ell_{+}-\epsilon, \ell_{+}\right]\right)-\log \mu\left(\left\{\ell_{+}\right\}\right)\right)=\infty .
\end{aligned}
$$

In a similar manner we have

$$
\begin{aligned}
\sigma_{m}: & =\int_{\left(0, \ell_{+}\right)} m((0, x]) \mathrm{d} x=\int_{0}^{\ell_{+}} \int_{0}^{x} \frac{\mu(\mathrm{d} y) \mathrm{d} x}{\int_{y}^{\ell_{+}}(u-y) \mu(\mathrm{d} u)}=\int_{0}^{\ell_{+}} \frac{\left(\ell_{+}-y\right) \mu(\mathrm{d} y)}{\int_{y}^{\ell_{+}}(u-y) \mu(\mathrm{d} u)} \\
& \geq \int_{0}^{\ell_{+}} \frac{\left(\ell_{+}-y\right) \mu(\mathrm{d} y)}{\int_{y}^{\ell_{+}}\left(\ell_{+}-y\right) \mu(\mathrm{d} u)}=\int_{0}^{\ell_{+}} \frac{\mu(\mathrm{d} y)}{\mu\left(\left[y, \ell_{+}\right]\right)}=\infty .
\end{aligned}
$$

Hence, by definition, $\ell_{+}$is a natural boundary for $\left(X_{t}\right)$ which can not be reached in finite time starting from a point $x<\ell_{+}$, see [10], Sections 5.11 and 5.16. 
Suppose $\mu\left(\left\{\ell_{+}\right\}\right)>0$. First note that $\ell_{+}$is a trap since $m\left(\left\{\ell_{+}\right\}\right)=\infty$ by the definition of $m$ in equation (3.1), and hence $\left(X_{t}\right)$ is absorbed in $\ell_{+}$upon reaching it. Further we have

$$
\begin{aligned}
\sigma_{m} & =\int_{\left(0, \ell_{+}\right)} \frac{\left(\ell_{+}-y\right) \mu(\mathrm{d} y)}{\int_{y}^{\ell_{+}}(u-y) \mu(\mathrm{d} u)} \leq \int_{\left(0, \ell_{+}\right)} \frac{\left(\ell_{+}-y\right) \mu(\mathrm{d} y)}{\int_{\frac{y+\ell_{+}}{2}}^{\ell_{+}}(u-y) \mu(\mathrm{d} u)} \\
& \leq 2 \int_{\left(0, \ell_{+}\right)} \frac{\mu(\mathrm{d} y)}{\mu\left(\left[\frac{y+\ell_{+}}{2}, \ell_{+}\right]\right)}<\infty .
\end{aligned}
$$

It follows that if $\ell_{+}$is the endpoint of a regular interval for $\left(X_{t}\right)$, i.e. if $m\left(\left(\ell_{+}-\epsilon, \ell_{+}\right)\right)>0$ for all $\epsilon>0$ then $\ell_{+}$is a regular or exit boundary and hence the process $\left(X_{t}\right)$ can reach $\ell_{+}$in finite time. Finally, if $\ell_{+}$is an isolated point in the support of $\mu$, i.e. if $\mu\left(\left(\ell_{+}-\epsilon, \ell_{+}\right]\right)=\mu\left(\left\{\ell_{+}\right\}\right)$for some $\epsilon>0$, then likewise it is an isolated absorbing point in the state space of $\left(X_{t}\right)$. It is easy to see that it can be reached in finite time with positive probability by considering $\left(X_{t}\right)$ reaching the point $\sup \left\{x \in \operatorname{supp}(\mu): x<\ell_{+}\right\}$and behaving thereafter.

\section{A MORE INTUitive AND DIRECT PROOF}

We have so far presented two methods of arriving at the representation (3.1) and Theorem 3.1. Both relied on deep probabilistic or analytic results and neither method appears to give a strong insight into why the result might be true. Consequently, one might want to have a more bare-handed proof, particularly if one wishes to generalise the result to other settings. Our goal in this section is to give a direct proof of Theorem 3.1, using a coupling and a construction of a martingale diffusion as a time-change of Brownian motion. The intuitive picture on which we base our proofs exploits the fact that we can write a time-changed martingale diffusion as a Brownian motion. In this picture, "locally", the process would appear to stop according to an exponential random variable, whose parameter would depend on the speed of the diffusion at that location; generalising this idea, we propose modelling the choice of an exponential stopping time by a Poisson random measure on $\mathbb{R}_{+} \times \mathbb{R}$, where points are placed with intensity $\mathrm{d} u m(\mathrm{~d} x)$, i.e. with more frequency in places where we expect to stop more often. Then we stop the process at a point $x$, if there is a point at $(u, x)$ in the Poisson random measure, and if the local time of the Brownian motion at $x$ reaches $u$, before the local time at any other $x^{\prime}$ reaches $u^{\prime}>0$ for some other point $\left(u^{\prime}, x^{\prime}\right)$ of the Poisson random measure. By comparing these stopping times $T^{m}$ derived from different Poisson random measures, we are able to prove a monotonicity result. This gives us a coupling argument from which we deduce the existence of a measure $m$ with the desired stopping distribution, i.e. $B_{T^{m}} \sim \mu$. Some simple calculations show that construction of a suitable generalised diffusion follows. This new insight then allows us to give an intuitive justification of the explicit formula (3.1). Observe that effectively we re-interpret the original problem as a new problem of finding a solution to the Skorokhod embedding problem (cf. [19]) in a given class of stopping times $T^{m}$.

We fix the underlying Brownian motion $\left(B_{t}\right)$ and the stopping times will be based on its local times $L_{t}^{x}$. We think about the behaviour of the process in the context of the curve of the local times $L_{t}^{x}$ of $B_{t}$ as $t$ is increasing. More specifically, define

$$
R_{t}=\left\{(u, x): L_{t}^{x}>u\right\}
$$

which is the set of points "inside" the local time curve. Now, given a measure $m(\mathrm{~d} x)$, we suppose $\Delta^{m}$ is a Poisson random measure on $\mathbb{R}_{+} \times \mathbb{R}$ with intensity measure $\mathrm{d} u m(\mathrm{~d} x)$, independent of the Brownian motion $B$. We allow $m(\mathrm{~d} x)$ to be infinite on some intervals. More precisely we assume that there exists a, possibly infinite, interval $I$, containing the origin, such that $m(\Gamma)<\infty$ for any compact set $\Gamma \subset I$ and that $m_{\mid I^{c}} \equiv \infty$. This agrees with equation (2.1). Formally the measure $\Delta^{m}$ decomposes into $\Delta^{m}=\tilde{\Delta}+\Delta_{\infty}$ where $\tilde{\Delta}$ is a Poisson random measure with intensity $\mathrm{d} u m_{\mid I}(\mathrm{~d} x)$ and $\Delta_{\infty}=\sum_{x \notin I} \delta_{(0, x)}$. We adopt this convention from now on.

We define the stopping time

$$
T^{m}=\inf \left\{t \geq 0: \Delta^{m}\left(R_{t}\right) \geq 1\right\}
$$




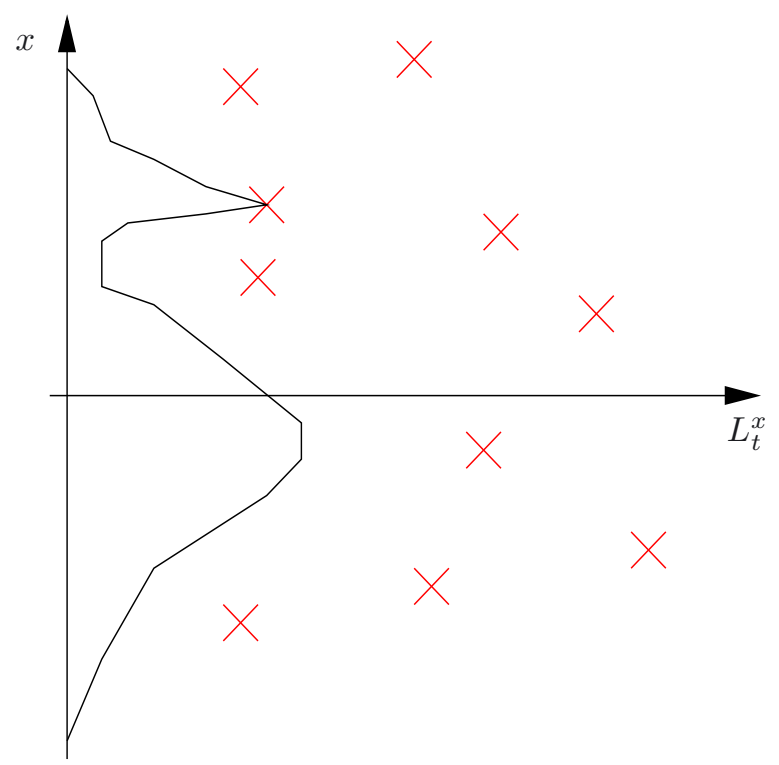

Figure 1. (Color online) The curve on the left represents the local time $L_{t}^{x}$ at time $t$. As time increases, the curve moves outwards. The crosses are distributed according to $\Delta$ and $T$ occurs the first time the local time curve hits such a point.

Figure 1 shows a graphical representation of the construction. The idea now is to construct $m$ such that $B_{T^{m}} \sim \mu$ and then time change with $A_{t}^{m}$ to obtain the desired generalised diffusion process as in equation (2.2). This is explained in the following theorem.

Theorem 5.1. Given a centred probability distribution $\mu$ on $\mathbb{R}$ there exists a measure $m$ such that $T^{m}$ is minimal $^{4}$ and embeds $\mu: B_{T^{m}} \sim \mu$.

Furthermore, if $\left(X_{t}^{m}\right)$ is a generalised diffusion given via equation (2.2) then the stopping time

$$
\tilde{T}^{m}=A_{T^{m}}^{-1}
$$

is exponentially distributed, independently of $X$, and $X_{\tilde{T}^{m}}=B_{T^{m}} \sim \mu$.

Remark 5.2. The above statement has two advantages in our opinion. Firstly, it provides an additional insight into the relation between stopping times for $X$ and $B$. Secondly, it can be proved using a fairly direct and elementary arguments. We note however that it is poorer than Theorem 3.1 which not only gives existence of $m$ but also the explicit formula (3.1).

Proof. We prove the first part of the theorem in two steps: in Step 1 we assume that $\mu$ has bounded support and in Step 2 we extend the result to arbitrary $\mu$ via a limiting procedure. Finally we prove the second part of the theorem.

Whenever no ambiguity is possible we suppress the superscripts $m$.

Part I. Step 1. We assume that $\mu$ has bounded support and denote by $\left[\ell_{-}, \ell_{+}\right]$the smallest closed interval with $\mu\left(\left[\ell_{-}, \ell_{+}\right]\right)=1$. Define the set

$$
S^{\mu}=\left\{m: \forall \Gamma \subset\left(\ell_{-}, \ell_{+}\right), \mathbb{P}\left(B_{T^{m}} \in \Gamma\right) \leq \mu(\Gamma)\right\}
$$

\footnotetext{
${ }^{4}$ Following [18], a stopping time $T$ is minimal if $S \leq T$ a.s. and $B_{S} \sim B_{T}$ imply $S=T$ a.s. Then if $B_{T}$ is a centred random variable, and $B_{0}=0$, minimality of $T$ is equivalent to the uniform integrability of $\left(B_{t \wedge T}, t \geq 0\right)$.
} 
We will now show that this set has non-trivial elements. Fix $\epsilon>0$ and let $m_{\epsilon}=\epsilon \mu$ on $\left(\ell_{-}, \ell_{+}\right)$and infinity elsewhere. We have then $T^{m_{\epsilon}} \leq H_{\ell_{-}} \wedge H_{\ell_{+}}$, where $H_{a}=\inf \left\{t: B_{t}=a\right\}$, and thus $\mathbb{E} L_{T^{m_{\epsilon}}}^{x} \leq 2 \frac{\ell_{+}\left|\ell_{-}\right|}{\ell_{+}-\ell_{-}}$. Let $\Gamma \subset\left(\ell_{-}, \ell_{+}\right)$and define:

$$
R_{t}^{\Gamma}=\left\{(u, x): L_{t}^{x}>u, x \in \Gamma\right\}
$$

Then we deduce:

$$
\begin{aligned}
\mathbb{P}\left(B_{T^{m_{\epsilon}}} \in \Gamma\right) & \leq \mathbb{P}\left(\Delta^{m_{\epsilon}}\left(R_{H_{\ell_{-}} \wedge H_{\ell_{+}}}^{\Gamma}\right) \geq 1\right) \\
& \leq \mathbb{E}\left[\Delta^{m_{\epsilon}}\left(R_{H_{\ell_{-}} \wedge H_{\ell_{+}}}^{\Gamma}\right)\right]=\epsilon \int_{\Gamma} \mathbb{E} L_{H_{\ell_{-}} \wedge H_{\ell_{+}}}^{y} \mu(\mathrm{d} y) \\
& \leq \frac{2 \epsilon \ell_{+}\left|\ell_{-}\right|}{\ell_{+}-\ell_{-}} \mu(\Gamma) .
\end{aligned}
$$

In consequence, for $\epsilon<\left(2 \ell_{+}\left|\ell_{-}\right| /\left(\ell_{+}-\ell_{-}\right)\right)^{-1}, m_{\epsilon} \in S^{\mu}$. We want to take the maximal element of $S^{\mu}$ and the following lemma describes the key property for our proof.

Lemma 5.3. Suppose that $m_{1}, m_{2} \in S^{\mu}$. Then the measure $e^{5} m=\max \left\{m_{1}, m_{2}\right\}$ is also an element of $S^{\mu}$.

The proof of the lemma, perhaps the most interesting element of the proof of Theorem 3.1, is postponed. Using the lemma we can conclude that there exists a maximal element $m^{\max } \in S^{\mu}$. We claim that $B_{T^{m} \max } \sim \mu$. Suppose the contrary and let $\nu \sim B_{T^{m} \max }$. As $m^{\max }$ is an element of $S^{\mu}, \nu$ is dominated by $\mu$ on $\left(\ell_{-}, \ell_{+}\right)$and with our assumption there exists $\Gamma \subset\left(\ell_{-}, \ell_{+}\right)$such that $\nu(\Gamma)<\mu(\Gamma)$. Let $f$ be the Radon-Nikodym derivative of $\nu$ with respect to $\mu$ on $\left(\ell_{-}, \ell_{+}\right)$. Then there exists an $\epsilon>0$ and $\Gamma^{\prime} \subseteq \Gamma$ such that $f<1-\epsilon$ on $\Gamma^{\prime}$ and $\mu\left(\Gamma^{\prime}\right)>0$. Let $m^{\prime}=m^{\max }+\gamma \mu \mathbf{1}_{\Gamma^{\prime}}$ with $\gamma=\epsilon\left(\ell_{+}-\ell_{-}\right) /\left(4 \ell_{+}\left|\ell_{-}\right|\right)$and let $\rho \sim B_{T^{m^{\prime}}}$. The measure $m^{\prime}$ involves extra stopping in $\Gamma^{\prime}$, when compared with $m$, so that necessarily there is less chance in stopping off $\Gamma^{\prime}$. Hence, $\rho \leq \nu \leq \mu$ on $\left(\ell_{-}, \ell_{+}\right) \backslash \Gamma^{\prime}$. Moreover, using arguments as above we see that $\rho \leq \nu+\epsilon / 2 \leq \mu$ on $\Gamma^{\prime}$ and thus $m^{\prime} \in S^{\mu}$ which contradicts maximality of $m^{\max }$. Finally, $T^{m^{\max }}$ is minimal since it is smaller than $H_{\ell_{-}} \wedge H_{\ell_{+}}$.

Part I. Step 2. Consider $\mu$ any centred probability measure and write $\ell_{-}, \ell_{+}$respectively for the lower and the upper bound of the support of $\mu$. We have just treated the case when both $\ell_{-}, \ell_{+}$are finite so we suppose that at least one of them is infinite. Let $a(n), b(n)$ be two sequences with $a(n) \searrow \ell_{-}, b(n) \nearrow \ell_{+}$, as $n \rightarrow \infty$, such that the measure

$$
\mu_{n}=\mu((-\infty, a(n)]) \delta_{a(n)}+\mu_{\mid(a(n), b(n))}+\mu([b(n), \infty)) \delta_{b(n)}
$$

is centred. This measure can be embedded using $T^{m_{n}}$ where $m_{n}=m^{\max }$ is the maximal element of $S^{\mu_{n}}$. Clearly $m_{k} \in S^{\mu_{n}}$ for all $k \geq n$ and thus $\left(m_{n}\right)$ is a decreasing sequence. It thus converges to a limit denoted $m=\inf _{n} m_{n}$, which is a measure (see e.g. Section III.10 in [6]).

Let $\Delta$ and $\tilde{\Delta}_{n}, n \geq 1$, be independent Poisson measures with intensities respectively $m$ and $\left(m_{n}-m_{n+1}\right)$, $n \geq 1$. Consider $\Delta_{n}=\Delta+\sum_{k \geq n} \tilde{\Delta}_{k}$ which is again a Poisson point measure with intensity $m+\sum_{k \geq n}\left(m_{k}-\right.$ $\left.m_{k+1}\right)=m_{n}$. With $T^{m_{n}}=\inf \left\{t: \Delta_{n}\left(R_{t}\right) \geq 1\right\}$ as previously, we have that $B_{T^{m_{n}}} \sim \mu_{n}$. Furthermore, as $\Delta_{n}(\Gamma) \geq \Delta_{n+1}(\Gamma) \geq \ldots$ we have that $T^{m_{n}} \leq T^{m_{n+1}} \leq \ldots$ so that $T^{m_{n}} \nearrow T=T^{m}$ as $n \rightarrow \infty$. To show that $T^{m}<\infty$ a.s. we recall that $\left|B_{t}\right|-L_{t}^{0}$ is a martingale. As $T^{m_{n}} \leq H_{a(n), b(n)}$ we have that

$$
\mathbb{E} L_{T^{m_{n}}}^{0}=\mathbb{E}\left|B_{T^{m_{n}}}\right| \leq \int_{-\infty}^{\infty}|x| \mu(\mathrm{d} x) .
$$

\footnotetext{
${ }^{5}$ See the proof of Lemma 5.3 for a detailed definition of $m$.
} 
The left hand side converges to $\mathbb{E} L_{T^{m}}^{0}$ which is thus finite and in particular $T^{m}<\infty$ a.s. Finally, since $m \in S^{\mu_{n}}$ for all $n$, the law of $B_{T^{m}}$ is dominated by $\mu$ on $\mathbb{R}$ and is thus simply equal to $\mu$. The uniform integrability of $\left(B_{t \wedge T^{m}}: t \geq 0\right)$ follows from standard arguments (e.g. Proposition 18 of [5]).

Part II. To show that $\tilde{T}^{m}$ is exponentially distributed, we recall the above definitions. Then for $t>s$ :

$$
\begin{aligned}
\mathbb{P}\left(\tilde{T}^{m} \geq t \mid \tilde{T}^{m} \geq s\right) & =\mathbb{P}\left(A_{T^{m}}^{-1} \geq t \mid A_{T^{m}}^{-1} \geq s\right) \\
& =\mathbb{P}\left(T^{m} \geq A_{t} \mid T^{m} \geq A_{s}\right) \\
& =\mathbb{P}\left(\Delta\left(R_{A_{t}-}\right)=0 \mid \Delta\left(R_{A_{s}-}\right)=0\right) \\
& =\mathbb{P}\left(\Delta\left(R_{A_{t}-} \backslash R_{A_{s}-}\right)=0\right) \\
& =\mathbb{P}\left(\Delta\left(R_{A_{t}} \backslash R_{A_{s}}\right)=0\right)
\end{aligned}
$$

where for the last equality we use the fact that local times are continuous in $t$. However, conditional on $B_{t}$, we know $\Delta\left(R_{A_{t}} \backslash R_{A_{s}}\right)$ is Poisson with parameter

$$
\int_{-\infty}^{\infty}\left(L_{A_{t}}^{x}-L_{A_{s}}^{x}\right) m(\mathrm{~d} x)=\Phi_{A_{t}}-\Phi_{A_{s}}=t-s
$$

where we used $\Phi_{A_{t}}=t$ since $\Phi_{t}$ is continuous. Clearly $X_{\tilde{T}^{m}}=B_{T^{m}}$. Finally a similar calculation to the ones above shows that $\mathbb{P}\left(\tilde{T}^{m}>t \mid \sigma\left(X_{u}: u \leq t\right)\right)=\mathbb{P}\left(\tilde{T}^{m}>t\right)$ and $\tilde{T}^{m}$ is independent of $X$. This completes the proof of Theorem 5.1.

Proof of Lemma 5.3. Note that the measure $m$ is well defined. More precisely let $m_{3}=m_{1}+m_{2}$ and $f_{1}$ and $f_{2}$ the Radon-Nikodym derivatives respectively of $m_{1}$ and $m_{2}$ with respect to $m_{3}$. The measure $m$ is defined via its Radon-Nikodym derivative $f=f_{1} \vee f_{2}$ with respect to $m_{3}$. Likewise, the measure $\underline{m}=\min \left\{m_{1}, m_{2}\right\}$ is well defined. We write $\nu^{m}$ for the law of $B_{T^{m} \wedge H_{a} \wedge H_{b}}$. Note that by construction $\nu \underline{\underline{m}}(\Gamma) \leq \nu^{m_{1}}(\Gamma) \leq \mu(\Gamma)$ and hence $\underline{m} \in S^{\mu}$.

Consider the signed measure $\left(m_{1}-m_{2}\right)$ and let $F_{1}$ be the support of its positive part and $F_{2}$ the support of its negative part. Then we may decompose $\Delta$ associated with $m$ into three independent Poisson Random Measures, $\Delta_{\wedge}$ with intensity $\mathrm{d} u \underline{m}(\mathrm{~d} x), \Delta_{1}$ with intensity $\mathrm{d} u\left(m_{1}(\mathrm{~d} x)-m_{2}(\mathrm{~d} x)\right) \mathbf{1}_{\left\{F_{1}\right\}}(x)$ and $\Delta_{2}$ with intensity $\mathrm{d} u\left(m_{2}(\mathrm{~d} x)-m_{1}(\mathrm{~d} x)\right) \mathbf{1}_{\left\{F_{2}\right\}}(x)$. We know that the stopping times generated by the measures $\Delta_{\wedge}+\Delta_{1}$ and $\Delta_{\wedge}+\Delta_{2}$ both lead to measures which are dominated by $\mu$ on $(a, b)$. We wish to deduce the same about $\Delta_{\wedge}+\Delta_{1}+\Delta_{2}$. We show this by considering the coupling implied by Figure 2. Given a set $\Gamma \subset(a, b)$, we need to show that $\nu^{m}(\Gamma) \leq \mu(\Gamma)$. However by considering $\Gamma \subseteq F_{1}$, it is clear that $\nu^{m}(\Gamma) \leq \nu^{m_{1}}(\Gamma)$ since adding adding extra marks according to $\Delta_{2}$ can only reduce the probability of stopping in $F_{1}$, as it will not produce any new "points" in this set. Similarly, for $\Gamma \subseteq F_{2}$, we will have $\nu^{m}(\Gamma) \leq \nu^{m_{2}}(\Gamma)$. Finally, for $\Gamma \subseteq(a, b) \backslash\left(F_{1} \cup F_{2}\right)$ we have $\nu^{m}(\Gamma) \leq \nu \underline{\underline{m}}(\Gamma)$. It now follows from $m_{1}, m_{2}, \underline{m} \in S^{\mu}$ that $m \in S^{\mu}$.

We have thus proved existence of a suitable measure $m$ such that the generalised diffusion $\left(X_{t}\right)$ associated to $m$ satisfies $X_{T} \sim \mu$ for an independent exponential time $T$. We have also seen that this is equivalent to finding $m$ such that $B_{T^{m}} \sim \mu$, where $T^{m}$ is stopping time defined in equation (5.1). We can use this new interpretation to recover the formula (3.1) for $m$, using an argument similar to that in Section 1.2. Indeed, from the construction of $T^{m}$, intuitively we have

$$
\mathbb{P}\left(B_{T^{m}} \in \mathrm{d} x\right)=m(\mathrm{~d} x) \times \mathbb{E}\left(\text { time spent in }\{x\} \text { by }\left(B_{t}: t \leq T^{m}\right)\right) .
$$




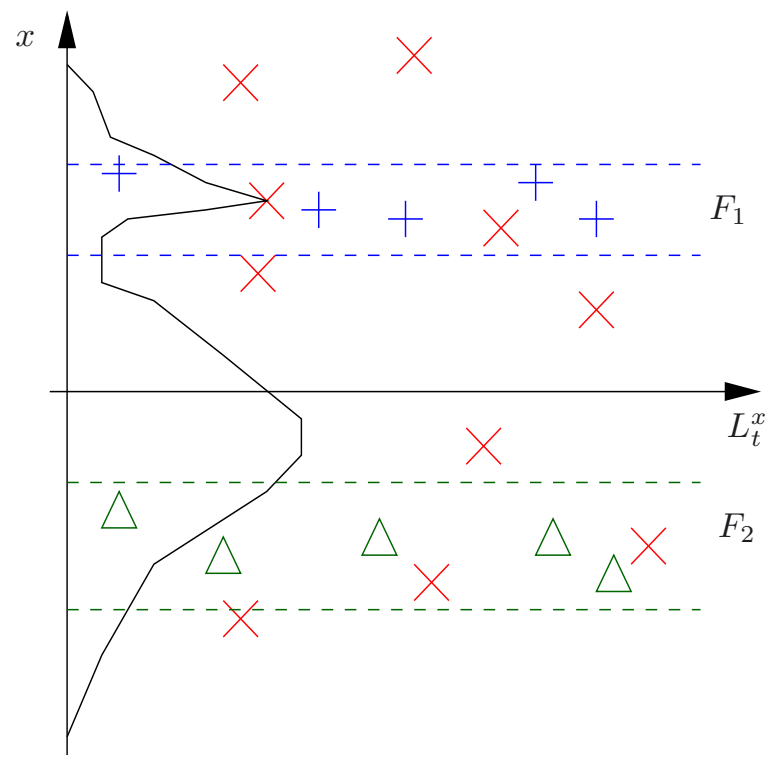

Figure 2. (Color online) Representation of the Poisson random measure $\Delta$ in terms of $\Delta_{\wedge}, \Delta_{1}$ and $\Delta_{2}$, represented by $\times,+$ and $\triangle$ respectively.

The time spent in $\{x\}$ by $\left(B_{t}: t \leq T^{m}\right)$ is simply $L_{T^{m}}^{x}$ and $\mathbb{E}\left[L_{T^{m}}^{x}\right]=\mathbb{E}\left|B_{T^{m}}-x\right|-|x|$. Hence, if we are to have $B_{T^{m}} \sim \mu$ we have to have

$$
\mu(\mathrm{d} x)=m(\mathrm{~d} x) \times\left(\int|x-y| \mu(\mathrm{d} y)-|x|\right)=m(\mathrm{~d} x) \times\left(u_{\mu}(x)-|x|\right),
$$

which is exactly equation (3.1).

Acknowledgements. Research partially supported by a Marie Curie Intra-European Fellowship at Imperial College London within the 6th European Community Framework Programme, and by the Oxford-Man Institute of Quantitative Finance.

\section{REFERENCES}

[1] J. Bertoin and Y. Le Jan, Representation of measures by balayage from a regular recurrent point. Ann. Probab. 20 (1992) 538-548.

[2] P. Carr, Local Variance Gamma. Private communication (2008).

[3] P. Carr and D. Madan, Determining volatility surfaces and option values from an implied volatility smile, in Quantitative Analysis of Financial Markets II, edited by M. Avellaneda. World Scientific (1998) 163-191.

[4] R.V. Chacon, Potential processes. Trans. Amer. Math. Soc. 226 (1977) 39-58.

[5] A.M.G. Cox, Extending Chacon-Walsh: minimality and generalised starting distributions, in Séminaire de Probabilités XLI. Lecture Notes in Math. 1934, Springer, Berlin (2008) 233-264.

[6] J.L. Doob, Measure theory. Graduate Texts Math. 143, Springer-Verlag, New York (1994).

[7] B. Dupire, Pricing with a smile. Risk 7 (1994) 18-20.

[8] H. Dym and H.P. McKean, Gaussian processes, function theory, and the inverse spectral problem. Probab. Math. Statist. 31. Academic Press (Harcourt Brace Jovanovich Publishers), New York (1976).

[9] D. Hobson, The Skorokhod Embedding Problem and Model-Independent Bounds for Option Prices, in Paris-Princeton Lectures on Mathematical Finance 2010, edited by R.A. Carmona, E. Çinlar, I. Ekeland, E. Jouini, J.A. Scheinkman and N. Touzi. Lecture Notes in Math. 2003, Springer (2010) 267-318. www.warwick.ac.uk/go/dhobson/

[10] K. Itô, Essentials of stochastic processes. Translations Math. Monographs 231, American Mathematical Society, Providence, RI, (2006), translated from the 1957 Japanese original by Yuji Ito. 
[11] L. Jiang and Y. Tao, Identifying the volatility of underlying assets from option prices. Inverse Problems 17 (2001) $137-155$.

[12] I.S. Kac and M.G. Kreın, Criteria for the discreteness of the spectrum of a singular string. Izv. Vyšs. Učebn. Zaved. Matematika 2 (1958) 136-153.

[13] F.B. Knight, Characterization of the Levy measures of inverse local times of gap diffusion, in Seminar on Stochastic Processes (Evanston, Ill., 1981). Progr. Probab. Statist. 1, Birkhäuser Boston, Mass. (1981) 53-78.

[14] S. Kotani and S. Watanabe, Kreı̆n's spectral theory of strings and generalized diffusion processes, in Functional analysis in Markov processes (Katata/Kyoto, 1981). Lecture Notes in Math. 923, Springer, Berlin (1982) 235-259.

[15] M.G. Kreĭn, On a generalization of investigations of Stieltjes. Doklady Akad. Nauk SSSR (N.S.) 87 (1952) 881-884.

[16] U. Küchler and P. Salminen, On spectral measures of strings and excursions of quasi diffusions, in Séminaire de Probabilités XXIII. Lecture Notes in Math. 1372, Springer, Berlin (1989) 490-502.

[17] D.B. Madan and M. Yor, Making Markov martingales meet marginals: with explicit constructions. Bernoulli 8 (2002) 509-536.

[18] I. Monroe, On embedding right continuous martingales in Brownian motion. Ann. Math. Statist. 43 (1972) $1293-1311$.

[19] J. Obłój, The Skorokhod embedding problem and its offspring. Prob. Surveys 1 (2004) 321-392.

[20] L.C.G. Rogers and D. Williams, Diffusions, Markov processes, and martingales, volume 2, Itô Calculus. Cambridge University Press, Cambridge, reprint of the second edition of 1994 (2000). 